\title{
Random demodulation for structural health monitoring excited by the five-cycle sine burst
}

\author{
Xing Li, Dong Liang*, Yishou Wang, Xinlin Qing, Hu Sun \\ School of Aerospace Engineering, Xiamen University, Xiamen 361005, China \\ 2458202237@qq.com
}

\begin{abstract}
Nowadays, the Structural Health Monitoring (SHM) has been paid more and more attention. The five-cycle sine burst is widely used as the exciting signal in SHM and the sensors' responded signals are analyzed to research the damage. In the sensor network, there will be many sensors which mean many responded signals will be sampled, restored and sometimes transferred. In the traditional way which is known as Nyquist sampling theorem, the sampling rate must be more than twice the highest rate of the original signal. In this way, the amount of data will be huge. As the result, the costs will be very expensive and the equipment may be huge and heavy, which is especially unaccepted in the aircraft. It is necessary to do some research to compress the signal. The Compressing Sensing (CS) theory provides new methods to compress the signals. The Random Demodulation (RD) is a specific method which can accomplish the physical implementation of CS theory. In this paper, according to the structure of RD, we chose some chips to build a RD system. And we did some experiments to verify the method through the system. We chose the Orthogonal Matching Pursuit (OMP) as the construct algorithm to recover the signal.
\end{abstract}

\section{Introduction}

SHM offers an online and real-time monitoring. It can discover the structural damage or fatigue timely and avoid the risk. However, in practical applications of large structural health monitoring, a large number of distributed sensors are usually adopted to monitor the big dimension structures. Hence, how to obtain a fast and accurate impact signal from the big data is an important problem for the damage assessments. According to the different functions of the system, there are two types of monitoring. One is active monitoring, the other is passive monitoring [1-2]. In this paper, the research is on the active monitoring.

Recently, the CS theory has been researched by many scholars. It has a lot of advantages on signal compressing, like independent acquisition and reconstruction, simple computation and good compression. So the CS has a great application prospects on SHM [3-5]. In this paper, we used the $\mathrm{CS}$ in the active monitoring, aiming to achieve a wide range of high-resolution data acquisition in large-scale structure quickly and efficiently. The research is about the five-cycle sine burst compressed by the RD system.

The paper has 7 parts. Next, Section 2 introduces the background knowledge of RD system. In Section 3, the signal used in SHM is introduced. Section 4 gives simulations. Section 5 is the experimental part. And section 6 analyses the experimental results. Finally, section 7 makes a conclusion for the paper. 


\section{Random demodulation (RD)}

According to the CS theory, if a signal can be expressed as a product of a vector and a martrix

$$
\boldsymbol{x}=\Psi \alpha \text {. }
$$

And the (1) can also be written as

$$
x(t)=\sum_{i=1}^{N} \alpha_{i} \Psi_{i}(t)
$$

If there is a few big coefficients in $\boldsymbol{\alpha}$, then the $x(t)$ is sparse in the basis of $\Psi$.And the $x(t)$ can be compressed and sampled at the same time by the method of global observation.

$\mathrm{RD}$ is a physical implementation of the CS. The structure of the RD is shown in Fig. 1.

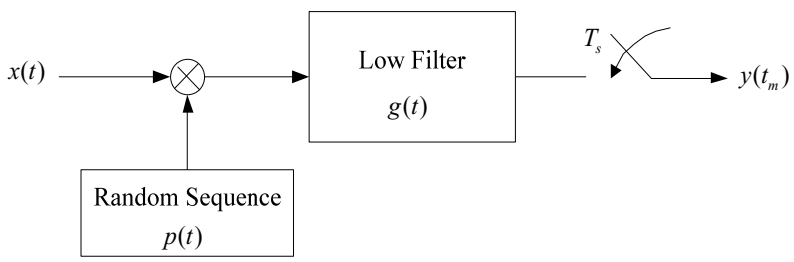

Fig. 1. The structure of RD

Its structure is simple and includes three main parts: mixing, filtering and sampling. The process expressed mathematically as

$$
y\left(t_{m}\right)=\left.\int_{-\infty}^{+\infty} x(\tau) p_{c}(\tau) h(t-\tau) \mathrm{d} \tau\right|_{t=t_{m}=m T_{s}}
$$

Substitute (2) into (3), the equation (3) is written as

$$
y\left(t_{m}\right)=\sum_{n=1}^{N} \alpha_{n} \int_{-\infty}^{+\infty} \Psi_{n}(\tau) p_{c}(\tau) h\left(m T_{s}-\tau\right) \mathrm{d} \tau=\sum_{n=1}^{N} \alpha_{n} v_{m, n}
$$

where $v_{m, n}=\int_{-\infty}^{+\infty} \Psi_{n}(\tau) p_{c}(\tau) h\left(m T_{s}-\tau\right) \mathrm{d} \tau$, where $h(t)$ is the unit impulse response of the filter. For the discrete signal, the output vector $\boldsymbol{y}$ is equivalent to multiply matrix $\boldsymbol{V}$ by the input vector $\alpha$ for the output vector $\boldsymbol{y}=V \boldsymbol{\alpha}$,

where $\boldsymbol{V}=\left[\begin{array}{cccc}v_{1}\left(t_{1}\right) & v_{2}\left(t_{1}\right) & \ldots & v_{N}\left(t_{1}\right) \\ v_{1}\left(t_{2}\right) & v_{2}\left(t_{2}\right) & \ldots & v_{N}\left(t_{2}\right) \\ \ldots & \ldots & \ldots & \ldots \\ v_{1}\left(t_{m}\right) & v_{2}\left(t_{m}\right) & \ldots & v_{N}\left(t_{m}\right)\end{array}\right]$. In this paper, the $\Psi$ is the Inverse Discrete Fourier Transform base.

$$
\Psi=\left[\begin{array}{ccccc}
1 & 1 & 1 & \cdots & 1 \\
1 & \bar{w} & \bar{w}^{2} & \cdots & \bar{w}^{N-1} \\
\vdots & \vdots & \vdots & \cdots & \vdots \\
1 & \bar{w}^{N-1} & \bar{w}^{2(N-1)} & \cdots & \bar{w}^{(N-1)(N-1)}
\end{array}\right]_{N \times N}
$$

where $\bar{w}=e^{\frac{2 \pi i}{N}}$.

There is a few big coefficients in $\boldsymbol{\alpha}$, so the solution of the underdetermined Equations $\boldsymbol{y}=V \boldsymbol{\alpha}$ problem can be converted into the minimum 0-norm problem:

$$
\hat{\boldsymbol{\alpha}}=\arg \min \|\boldsymbol{\alpha}\|_{0}, \text { s.t. } \boldsymbol{y}=\boldsymbol{V} \boldsymbol{\alpha} .
$$

To solve the equation (5), the orthogonal matching tracking algorithm (OMP) is chosen, because the speed of the reconstruction is fast[6-13].

\section{The narrowband waves used in SHM}

Recently, the five-cycle sine burst is widely used as the exciting signal in SHM. It is a narrow-band signal which can be expressed as

$$
f_{a}(t)=A \sin \left(2 \pi f_{c} t\right)\left(1-\cos \left(\frac{2 \pi f_{c} t}{5}\right)\right)
$$

where A denotes the amplitude and $f_{c}$ represents the central frequency of the signal[14]. The exciting signal is the first cycle from the beginning of the $\mathrm{x}$-axis of the $f_{a}(t)$. The rest is 0 . The Fig. 2 shows the exciting signal.

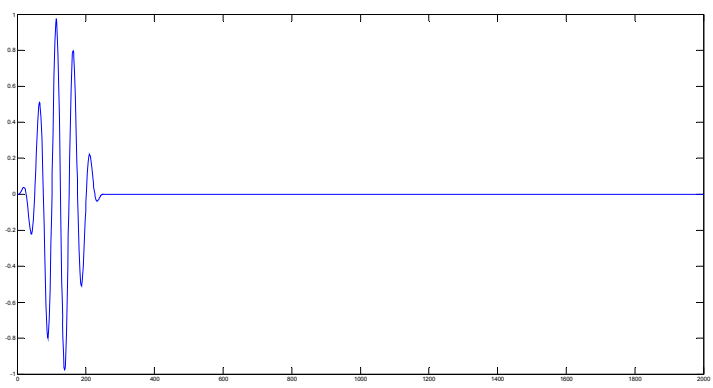

Fig 2. The five-cycle sine burst 


\section{Simulation}

\subsection{The simulation instructions}

The simulation is done by the MATLAB. The random sequence produced by function $\operatorname{randsrc}(\mathrm{N}, 1)$ which can produce a sequence that is N-row and 1 column with value of \pm 1 . The Butterworth is selected as the type of the filter using three functions, buttord, butter and buttap.

In the simulation, we use the Signal-Noise Ratio (SNR) to evaluate the effect of reconstruction. The SNR expressed as

$$
S N R=20 \square \log _{10} \frac{\|x\|_{2}}{\|x-\hat{x}\|_{2}},(7)
$$

where

$$
\|\bullet\|_{2} \quad \text { is } \quad \text { defined }
$$

as

$\|x\|_{2}=\sqrt{\left(\left|x_{1}\right|^{2}+\left|x_{2}\right|^{2}+\cdots+\left|x_{n}\right|^{2}\right)}, \quad \hat{x} \quad$ is the reconstructed signal while $x$ is the original signal.

The simulations are about sin waves and the five-cycle sine burst. In the simulation, the sampling rate of generating signal is $100 \mathrm{~K}$, so the sampling rate of the digital filter is $100 \mathrm{~K}$ too. We stipulate the reconstruction is successful when the SNR is higher than or equal to $15 \mathrm{~dB}$. The probability of successful reconstruction is another factor to evaluate the result and defines as the ratio of successful times and total times. And the compression ratio is 10 .

\subsection{The results}

The results of sin waves are very good and the SNR is very high. And the frequency of the signal reconstructed well can be very high, nearly the half of the sampling rate. The results are shown in Fig. 3.

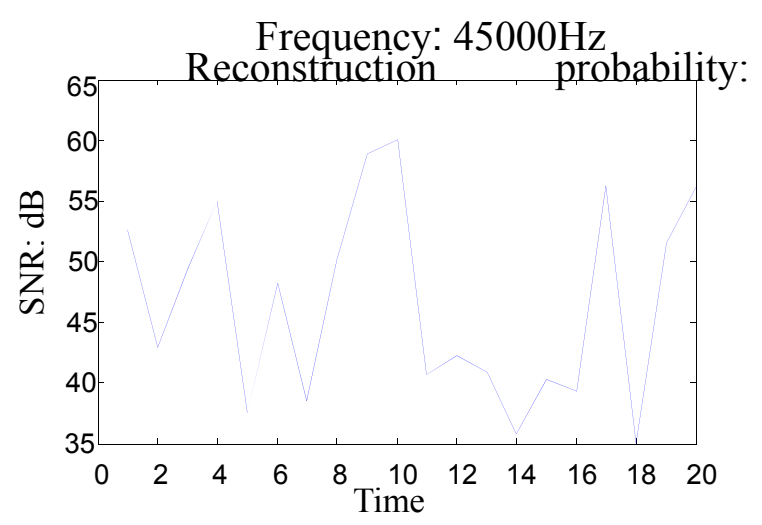

Fig. 3 The reconstructed result of sine waves

However, the results are not so good for the five-cycle sine burst. As the Fig. 4 shows, as the frequency becomes increased, the SNR and the reconstruction probability are decreased. When the frequency is $2000 \mathrm{~Hz}$, the reconstruction probability is even 0 . The relation between the frequency and the reconstruction probability is shown in Table. 1.

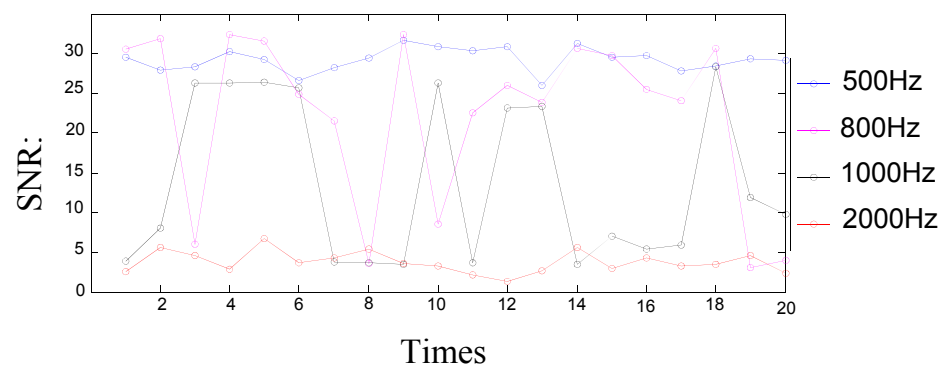

Fig. 4 The frequency and the SNR when the sampling rate is $100 \mathrm{~K} \mathrm{~Hz}$

Table 1. The frequency and the reconstruction probability when the sampling rate is $100 \mathrm{~K}$

\begin{tabular}{|l|l|l|l|l|}
\hline frequency $(\mathrm{Hz})$ & 500 & 800 & 1000 & 2000 \\
\hline $\begin{array}{l}\text { reconstruction } \\
\text { probability }(\%)\end{array}$ & 100 & 75 & 40 & 0 \\
\hline
\end{tabular}

The results show that the five-cycle sine burst has frequency limits on using in the RD compared to the sine waves. The reason may be the sparsity of signals in the Inverse Discrete Fourier Transform base.

There is only one nonzero factor in the spectrum of the sine waves. But in the spectrum of the five-cycle sine burst, it's a limited band. As shown in Fig. 5. 


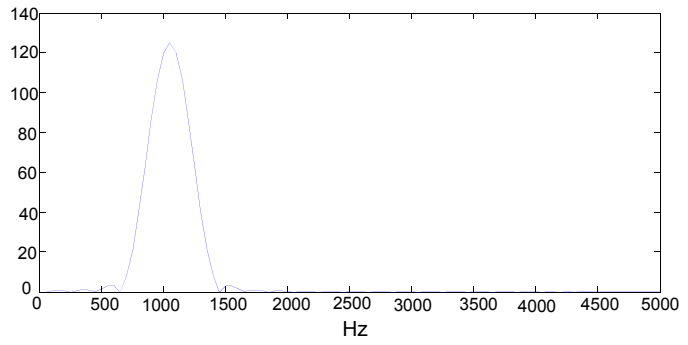

Fig. 5 The spectrum of the five-cycle sine burst

However, the low frequency can be reconstructed well, like $500 \mathrm{~Hz}$ and $800 \mathrm{~Hz}$. The frequency is higher, the results are worse. The reason is that as the frequency increased, the nonzero width of spectrum is wider, as the Fig. 6 shows.

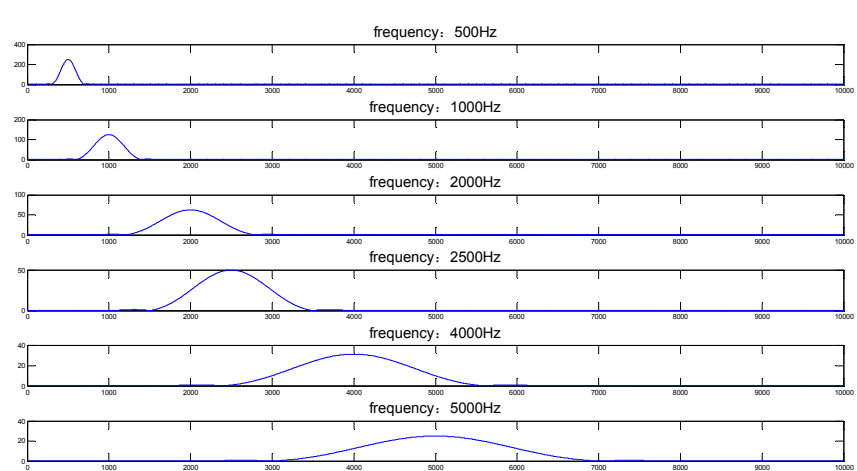

Fig. 6 The spectrum of different frequency of the five-cycle sine burst

According to the CS theory, obviously, there are less nonzero factors in the spectrum, the reconstructed results will be better. However, the sparsity is relative, but it is not to total points of signal, according to the simulation, it's to the sampling rate.

If we change the sampling rate of generating signal to $1000 \mathrm{KHz}$, the results' frequency increases 10 times too. The results are shown in the Fig. 7 and Table 2.

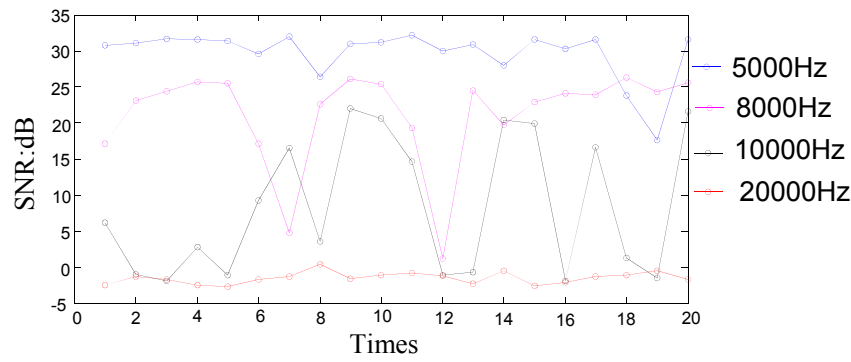

Fig. 7 The frequency and the SNR when the sampling rate is $1000 \mathrm{~K}$
Table 2. The frequency and the reconstruction probability when the sampling rate is $1000 \mathrm{~K}$

\begin{tabular}{|l|l|l|l|l|}
\hline Frequency (Hz) & 5000 & 8000 & 10000 & 20000 \\
\hline $\begin{array}{l}\text { Reconstruction } \\
\text { probability (\%) }\end{array}$ & 100 & 90 & 35 & 0 \\
\hline
\end{tabular}

It seems that the ratio of frequency and the sampling rate of generating signal must be in a specific range, the results will be good.

What's more, the cutoff frequency of the filter can influence the results. When the signal's frequency increases, increasing the cutoff frequency from the low frequency's cutoff frequency is helpful. But it's not positive correlative. The cutoff frequency must be suitable.

\section{Experiments}

\subsection{Experiment system}

We set up a system to put the RD in the active monitoring system. The structure is shown in Fig. 8.

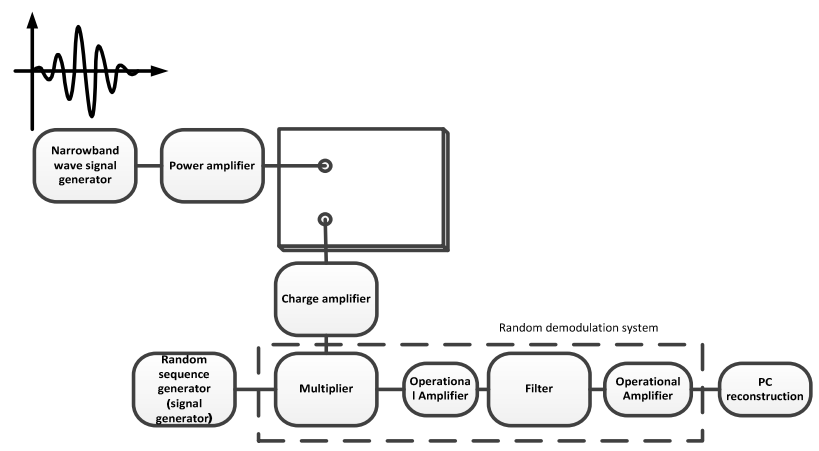

Fig. 8 The RD in the active monitoring system

As the Fig shows, the five-cycle sine burst is amplified by the power amplifier, then to one PZT sensor. The other sensor receives the reflected signal and is through the charge amplifier. Then the signal enters into the RD system, compressed, sampled and reconstructed.

\subsection{The method of experiment}

The reponse signal of sensor has many reflected waves, however, upon most occasions, only the 
first main response five-cycle sine burst wave is used in SHM. As shown in Fig. 9.

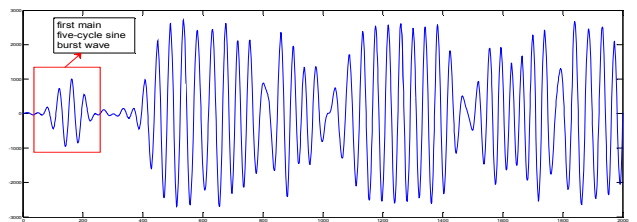

Fig. 9 The reponse signal of sensor

And the first main wave is the same as the exciting wave. For simplicity, firstly, we used the five-cycle sine burst produced by the signal generator instead of the response signal of sensor as the original signal to verify the method, as shown in Fig. 10 .

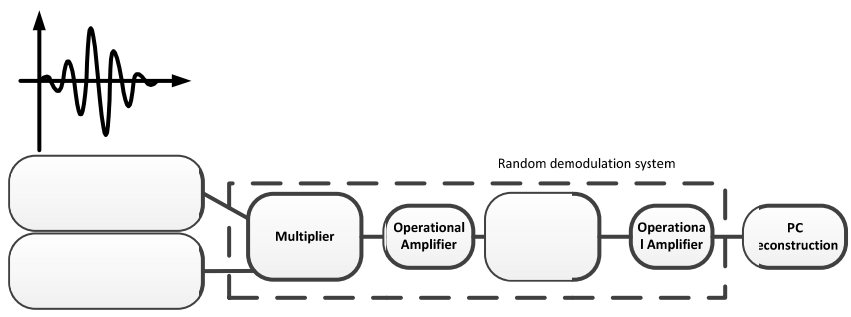

Fig. 10 The experimental system

The five-cycle sine burst is generated by NI PXIe-5442 wave generator; the random sequence is generated by NI PXIe-6115 and the sampling is done by NI PXIe-5122. The three cards are all in a chassis and transferred by the LabVIEW program. And the OMP algorithm is done by MATLAB and embedded in the LabVIEW program.

\subsection{The design of RD system}

The AD633 is used as the multiplier and the MAX275 is used as the filter. The operational amplifier after multiplier is OPA627, after filter is MAX44241.

The $\mathbf{m}$ sequence is chosen as the random sequence. m sequence is referred to as the longest linear feedback shift register sequence. It is a pseudo-random binary sequence and its magnitude approximately randomly jumps to 1 or -1 , which make its frequency spectrum looks similar to the broadband noise spectrum and cover almost the entire frequency axis. The $\mathbf{m}$ sequence is easily produced, regularity and has many excellent performance.

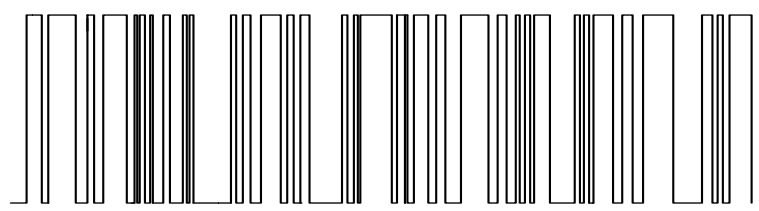

Fig. $11 \mathrm{~m}$ sequence

\section{Results of experiments and analysis}

\section{1 results}

The results of experiments' SNR are worse than the simulation. However, the reconstruction probability is similar to the simulations. Some results are shown in Fig. 12.

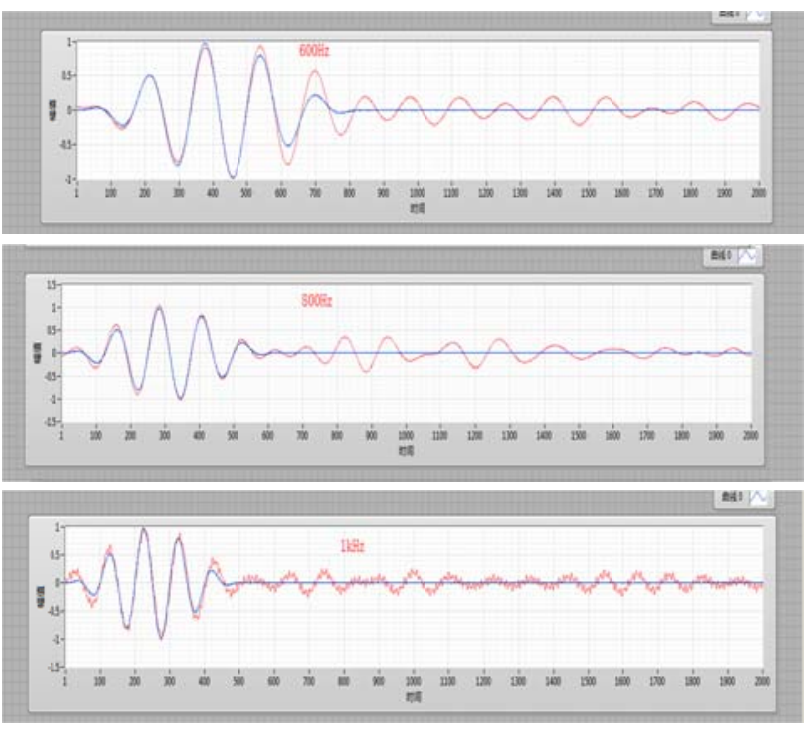

Fig. 12 The results of the experiments: the red is reconstructed signal, the blue is original one

As the results shown, the reconstructed signal has the roughly outline of the original signal. But there are still some differences in the details. Those differences in detail result in the lower SNR compared to the simulations. One important reason is the unit impulse response of the RD system.

\subsection{The unit impulse response}

In the experiments, we used the unit impulse response of the RD system rather than the filter in the simulation. And the unit impulse response has a great influence on the reconstructed results [15]. 
In this paper, we use the $\mathbf{m}$-sequences for impulse response measurement. There are many noises in the impulse in one measurement. So we measure and calculate several times to get its average. There is less noise in the impulse response, the reconstructed results will be better.

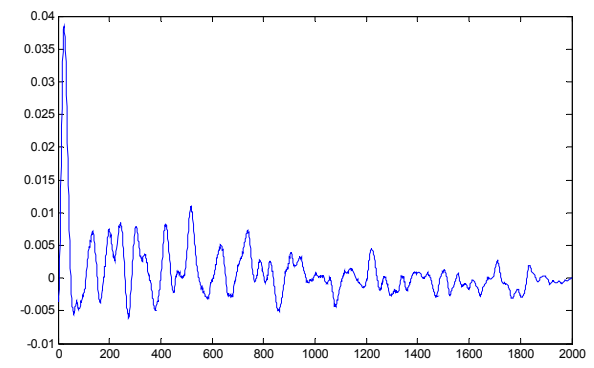

(a)

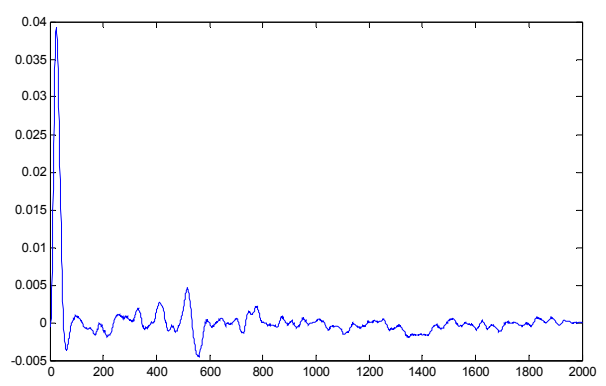

(b)

Fig. 13 The unit impulse response: the (a) is measured by once, the (b) is the average measured by ten times.

\subsection{The other reasons}

The noise in the unit impulse response is influenced by the chips, generator or sampling. The chips may produce the noise when they work. And there may be some mistakes in generating $\mathbf{m}$ sequence and sampling the output signals of the system. Those noise and mistakes result in the noise in the unit impulse response. What's more, the system may have some offsets. All those nonideal factors will lead the mistakes in the reconstructed results.

\section{Conclusion}

In this paper, we have done some research on using $\mathrm{RD}$ system to compress and sample the five-cycle sine burst. For now, the frequency of the five-cycle sine burst that can be well reconstructed is relatively low. Then we analyzed the reason of the problem. The main reason is that the five-cycle sine burst's spectrum is a limited band and as the frequency increases, the spectrum is wider. In the experiments, we found some factors that influence the practical application of the RD. The unit impulse response matters a lot and the accuracy of instruments and chips affects the results too.

\section{Acknowledgements}

This work is supported by the National Natural Science Foundation of China (Grant no. 51405409) and the Fundamental Research Funds for the Central Universities.

\section{References}

[1] K. Diamanti, C. Soutis, Structural health monitoring techniques for aircraft composite structures, Progress in Aerospace Sciences 46 (8) (2010) 342-352.

[2] Qing X, Beard S, Ikegami R, Chang F-K, Boller C. Aerospace Applications of SMART Layer Technology[M]. Encyclopedia of Structural Health Monitoring, Wiley, 2009.

[3] Alessandro Perelli, Sevan Harput, Luca De Marchi and Steven Freear. Frequency Warping Compressive Sensing for Structural Monitoring of Aircraft Wing[J], IEEE Transactions on Ultrasonics, Ferroelectrics, and Frequency Control, Vol: 60, pp.2089-2097, October. 2013.

[4] Yuan Mei, Wang Shujuan, Dong Shaopeng and Pang Zhuo. "Reconstruction of Undersampled Damage Monitoring Signal Based on Compressed Sensing". Guidance, Navigation and Control Conference (CGNCC), 2014 IEEE Chinese, Yantai, China, 2014, pp.2243-2248.

[5] Ju-min Zhao, Ding Feng, Deng-ao Li, and Bao-feng Zhao, "Power-free Structural Health Monitoring via compressive sensing", Computing and Communications Conference (IPCCC), 2015 IEEE 34th International Performance, Nanjing, China, 2015.

[6] D. Karampoulas , S.M.Kouadri , L.S.Dooley, "A 
Novel Precolouring-Random Demodulator Architecture for Compressive Spectrum Estimation”, Intelligent Signal Processing Conference 2013 (ISP 2013), IET, London, UK, 2013.

[7] Tamer Ragheb, Jason N. Laska, Hamid Nejati and Sami Kirolos, "A Prototype Hardware for Random Demodulation Based Compressive Analog-to-Digital Conversion", 51st Midwest Symposium on Circuits and Systems, Knoxville, TN, USA, 2008.

[8] Ning Fu, Pingfan Song, Peizhuo Liu, and Jingchao Zhang, "Boost the Efficiency of Spectrum Sensing Using Synchronized Random Demodulation", 19th International Conference on Digital Signal Processing, Hong Kong, China, 2014, pp.525-530.

[9] Ning Fu, Tingting Yao, Hongwei Xu, "COMPRESSIVE BLIND SOURCE RECOVERY WITH RANDOM DEMODULATION", 22nd European Signal Processing Conference (EUSIPCO), Lisbon, Portugal, 2014, pp.746-750.

[10] E. Astaiza, H. F. Bermúdez, W. Y. Campo, "Efficient Wideband Spectrum Sensing Based on Compressive Sensing and Multiband Signal Covariance", IEEE Latin America Transactions, vol:15, pp.393-399, 2017

[11] Smaili S, Massoud Y, "Accurate and efficient modeling of random demodulation based compressive sensing systems with a general filter", Circuits and Systems (ISCAS), 2014 IEEE International Symposium on. IEEE, 2014, pp. 2519-2522.

[12] Kirolos S, Laska J, Wakin M, et al, "Analog-to-information conversion via random demodulation", Design, Applications, Integration and Software, 2006 IEEE Dallas/CAS Workshop on. IEEE, 2006, pp.71-74.

[13] Ning Fu, Pingfan Song, Jingchao Zhang and Ying Liu, "A Random Demodulation Hardware System with Automatic Synchronization Function", IEEE International Instrumentation and Measurement Technology, Minneapolis, MN, USA, 2013, pp.1554-1558.

[14] Shan S, Qiu J, Zhang C, et al. Multi-damage localization on large complex structures through an extended delay-and-sum based method[J]. Structural Health Monitoring, 2016, 15(1): 50-64.

[15] Pawel J. Pankiewicz, Thomas Arildsen, Torben
Larsen, "SENSITIVITY OF THE RANDOM DEMODULATION FRAMEWORK TO FILTER TOLERANCES", Signal Processing Conference, European, 2011, pp.534-538. 